OPEN ACCESS

Edited by:

Mohiuddin Md. Taimur Khan,

Washington State University, USA

Reviewed by:

M. Jahangir Alam,

University of Houston, USA

Nehreen Majed,

University of Asia Pacific, Bangladesh

*Correspondence:

Munirul Alam

munirul@icddrb.org

Specialty section:

This article was submitted to

Environmental Health,

a section of the journal

Frontiers in Public Health

Received: 13 October 2016

Accepted: 19 December 2016

Published: 09 January 2017

Citation:

Johura FT, Parveen R, Islam A

Sadique A, Rahim MN, Monira S,

Khan AR, Ahsan S, Ohnishi M,

Watanabe H, Chakraborty $S$,

George CM, Cravioto A, Navarro A,

Hasan B and Alam M (2017)

Occurrence of Hybrid Escherichia coli

Strains Carrying Shiga Toxin and

Heat-Stable Toxin in Livestock of

Bangladesh.

Front. Public Health 4:287.

doi: 10.3389/fpubh.2016.00287

\section{Occurrence of Hybrid Escherichia coli Strains Carrying Shiga Toxin and Heat-Stable Toxin in Livestock of Bangladesh}

\author{
Fatema-Tuz Johura', Rozina Parveen', Atiqul Islam ${ }^{1}$, Abdus Sadique', Md Niaz Rahim', \\ Shirajum Monira', Anisur R. Khan'2, Sunjukta Ahsan', Makoto Ohnishi', Haruo Watanabe', \\ Subhra Chakraborty ${ }^{4}$, Christine M. George ${ }^{4}$, Alejandro Cravioto' ${ }^{1}$, Armando Navarro ${ }^{5}$, \\ Badrul Hasan ${ }^{6}$ and Munirul Alam ${ }^{1 *}$ \\ ${ }^{1}$ International Center for Diarrheal Disease Research, Bangladesh (icddr,b), Dhaka, Bangladesh, ${ }^{2}$ Department of \\ Microbiology, University of Dhaka, Dhaka, Bangladesh, ${ }^{3}$ National Institute of Infectious Diseases, Tokyo, Japan, ${ }^{4}$ Johns \\ Hopkins Bloomberg School of Public Health, Baltimore, MD, USA, ${ }^{5}$ Department of Public Health, Faculty of Medicine, \\ Universidad Nacional Autónoma de Mexico, Mexico City, Mexico, ${ }^{6}$ Infectious Disease, Department of Medical Sciences, \\ Uppsala University, Uppsala, Sweden
}

Shiga toxin-producing Escherichia coli (STEC) and enterotoxigenic E. coli (ETEC) are important causes of diarrhea in humans and animals worldwide. Although ruminant animals are the main source of STEC, diarrhea due to this pathotype is very low in Bangladesh where ETEC remains the predominant group associated with childhood diarrhea. In the present study, E. coli strains $(n=35)$ isolated from Bangladesh livestock (goats, sheep, and cattle) and poultry (chicken and ducks) were analyzed for the presence of major virulence factors, such as Shiga toxins (STX-1 and STX-2), heat-labile toxin, and heat-stable toxins (STa and STb). Multiplex polymerase chain reaction results revealed $23(66 \%)$ E. coli strains to be virulent possessing either sta $(n=5)$, stx (stx1, $n=8$; stx2, $n=2)$, or both $(n=8)$ genes in varying combinations. Thirty-four percent $(8 / 23)$ of strains from livestock were hybrid type that carried both stx (either stx 1 or stx2) and ETEC-specific enterotoxin gene sta. Serotyping results revealed that the ETEC strains belonged to five serotypes, namely O36:H5, O174:H-, O152:H8, O109:H51, and O8:H21, while the STEC-producing strains belonged to serotypes O76:H19 $(n=3)$, O43:H2 $(n=2)$, O87:H16 $(n=2), \mathrm{OR}: \mathrm{H} 2(n=1), \mathrm{O} 110: \mathrm{H} 16(n=1)$, and O152:H8 $(n=1)$. The STEC-ETEC hybrid strains belonged to serotypes O76: $\mathrm{H} 19(n=3), \mathrm{O} 43: \mathrm{H} 2$ $(n=2), \mathrm{O} 87: \mathrm{H} 16$, OR:H2, and O152:H8. Forty percent (2/5) of the ETEC and 20\% (2/10) of the STEC strains were multidrug resistant with the highest drug resistance (50\%) being found in the hybrid strains. Molecular fingerprinting determined by pulsed-field gel electrophoresis and cluster analyses by dendrogram revealed that, genetically, STEC-ETEC hybrid strains were highly heterogeneous. Multidrug-resistant E. coli STEC-ETEC hybrid strains in domesticated animals pose a public health threat for humans in Bangladesh.

Keywords: Shiga-toxin, enterotoxin, livestock, hybrid, multidrug resistant, PFGE 


\section{INTRODUCTION}

Escherichia coli is commonly regarded as a non-pathogenic beneficial inhabitant of the gastrointestinal tract but several pathogenic strains acquired specific virulence factors that are accountable for a variety of intestinal and extra intestinal diseases, including diarrhea, acute inflammation, hemorrhagic colitis, urinary tract infections, septicemia, and neonatal meningitis (1-4). Based on virulence traits, major diarrheagenic E. coli pathogroups include shigatoxigenic E. coli, enterotoxigenic E. coli (ETEC), enteropathogenic E. coli, enteroinvasive E. coli, and enteroaggregative E. coli (EAEC) (3). Shiga toxinproducing E. coli (STEC) is a pathogen of significant public health concern and infection by this strain can result in a spectrum of outcomes ranging from asymptomatic carriage to severe diarrhea, as well as bloody diarrhea and hemolyticuremic syndrome (HUS) (5). The disease causing ability of STEC is associated with the production of phage-encoded Shiga-like toxins (Stx), which are classified into two major families, Stx 1 and Stx2 (encoded by stx 1 and stx 2 genes) on the basis of toxin neutralization assays and sequence analysis of $s t x$ genes. ETEC is the leading cause of diarrhea in travelers from industrialized countries and children living in low-income countries. ETEC bacteria are largely defined by the presence of the plasmid-encoded heat-labile (LT) and/or heat-stable (STa/STb) toxins (3).

Ruminants, especially cattle, sheep, and goat are the main source of STEC for humans and play an important role in the epidemiology of human infections (6). Meat contamination can facilitate transmission of pathogenic E. coli occurring through unhygienic slaughtering and poor food handling. Screening and characterization of animal STEC helps to identify the origin of human STEC infections. Despite the fact that over 400 STEC serotypes have been identified, only a subset of these have been correlated to human illness (7). Most outbreaks of hemorrhagic colitis and HUS have been associated with STEC O157 strains (5). There are outbreaks reported that are caused by non-O157 (8); infections caused by some non-O157 serotypes have also been frequently associated with severe illness in humans. In some geographic areas, STEC non-O157 strains are more commonly isolated than STEC O157 strains from persons with diarrhea or HUS (9). Antimicrobial resistance patterns may be an additional epidemiological marker for surveys of non-O157 SETC (10).

In human, ETEC strains are associated with one of the most frequent (sometimes fatal) causes of childhood diarrhea in the developing countries and an important causative agent of traveler's diarrhea (3). ETEC is also a major cause of severe diarrheal disease in suckling and weanling animals (11). ETEC infections are transmitted through the fecal-oral route. Exposure to ETEC usually comes from contaminated food and drinking water (3). A close genetic relationship has been found between ETEC strains belonging to certain serotypes with diarrhea, which suggest that serotype analysis can be coupled with genetic typing to study strain clustering in epidemiologic and pathogenic studies. To assess the potential public health risk posed by ETEC and STEC, the detection of virulence factors, such as heat-stable toxin a
$(S T a)$, heat-stable toxin b $(S T b)$, heat-labile toxin $(L T)$, stxl, and stx2, is recommended (12-16).

Shigatoxigenic E. coli and other diarrheagenic E. coli are capable of acquiring virulence and related genes via horizontal gene transfer leading to the development of pathogroups different from the pre-existing ones (17). Such divergent pathogroups are often reported using different terminology such as "hybrid" (18), "blended virulence profiles," and "virulence combination" (19). Several studies have reported coexisting STEC- and ETECassociated virulence genes in E. coli strains of human, animal, and environmental origins $(17,20-25)$. The notorious sprout-borne outbreak strain O104:H4 in Germany possessed EAEC- and STEC-associated virulence genes (18) pointing to the possibility of extremely pathogenic strains emerging over a short time period (26).

In Bangladesh, the prevalence of STEC in hospitalized patients with diarrhea is very low (0.5\%) (27), but it was somewhat higher among community cases with diarrhea (1.9\%) (27). In Bangladesh, the predominant group of E. coli associated with childhood diarrhea is ETEC, which accounts for approximately $20 \%$ of all diarrheal cases (28). Animals seem to be the potential carrier for pathogenic $E$. coli but there is little data available concerning animal reservoirs, epidemiology, human pathogenicity, and drug resistance profiles of STEC and ETEC in Bangladesh. In the present study, we report the existence of multidrug-resistant E. coli strains belonging to STEC, ETEC, and a newly emerged STEC-ETEC hybrid in domesticated animals, which pose a potential public health threat for humans in Bangladesh.

\section{MATERIALS AND METHODS}

\section{Sample Collection, Isolation, and Identification of E. coli}

Rectal (livestock) and cloacal (bird) samples were collected from a total of 35 different livestock [goat $(n=19)$, sheep $(n=9)$, cattle $(n=2)$ ] and poultry [chicken $(n=3)$ and duck $(n=2)$ ] between June and August 2007 from the district of Mymensingh, Bangladesh. Sterile cotton swabs were used for the collection of rectal and cloacal samples. After collection, the cotton swabs were dipped into Carry-Blair media and stored in a cool box $\left(4^{\circ} \mathrm{C}\right)$. Immediately after sampling, all samples were transported to the laboratory for analysis. All samples were streaked onto MacConkey agar (Difco), and the plates were incubated overnight at $37^{\circ} \mathrm{C}$. Bright pink lactose-fermenting colonies were selected as presumptive E. coli. These colonies were again grown on eosin methylene blue agar to examine for the production green colonies with a metallic sheen. One presumptive colony per sample was identified by biochemical tests, as described before (29).

\section{Polymerase Chain Reaction (PCR) Amplification of Virulence Genes}

DNA was extracted from isolated colonies suggestive of E. coli, as described previously (30). The samples were then subjected to molecular analysis of ETEC (sta, stb, elt) and STEC (stx1, stx2) virulence genes using multiplex PCR. The PCR assay conditions are described in Table 1. Each amplification was conducted in a 
TABLE 1 | Primer sequences for STa, STb, LT, Stx1, and Stx2 genes, binding temperature, size of the amplification product, and reference of each primer used.

\begin{tabular}{|c|c|c|c|c|}
\hline Primers & Sequence & Annealing temperature $\left({ }^{\circ} \mathrm{C}\right)$ & $\begin{array}{l}\text { Size of the amplification } \\
\text { product (bp) }\end{array}$ & Reference \\
\hline STa & $\begin{array}{l}\text { TCC CCT CTT TTA GTC AGT CAA CTG } \\
\text { GCA CAG GCA GGA TTA CAA CAA AGT }\end{array}$ & 57 & 163 & Ngeleka et al. (12) \\
\hline STb & $\begin{array}{l}\text { GCA ATA AGG TTG AGG TGA T } \\
\text { GCC TGC AGT GAG AAA TGG AC }\end{array}$ & & 368 & Lortie et al. (13) \\
\hline$L T$ & $\begin{array}{l}\text { TTA CGG CGT TAC TAT CCT CTC TA } \\
\text { GGT CTC GGT CAG ATA TGT GAT TC }\end{array}$ & & 275 & Furrer et al. (14) \\
\hline Stx 1 & $\begin{array}{l}\text { AGA GCG ATG TTA CGG TाT G } \\
\text { TTG CCC CCA GAG TGG ATG }\end{array}$ & 55 & 388 & Jackson et al. (15) \\
\hline Stx2 & $\begin{array}{l}\text { TGG GTT TIT CTT CGG TAT C } \\
\text { GAC ATT CTG GTT GAC TCT CTT }\end{array}$ & & 807 & Jackson et al. (16) \\
\hline
\end{tabular}

volume of $20 \mu \mathrm{l}$ containing the following reagents: $1 \mu \mathrm{l}$ template DNA, $0.5 \mu \mathrm{l}$ dNTP (Invitrogen, Carlsbad, CA, USA) solution $\left(10 \mathrm{mmol}^{-1}\right), 1.0 \mu \mathrm{l}$ of each primer at $5 \mathrm{pmol} \mathrm{l}^{-1}, 0.6 \mu \mathrm{Taq}$

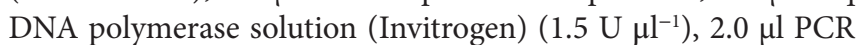
reaction buffer solution $(10 \times)$ with $0.8 \mu \mathrm{MgCl}_{2}\left(50 \mathrm{mmol} \mathrm{l}{ }^{-1}\right)$, and presterilized ultrapure water (Milli-Q) to $20 \mu \mathrm{l}$. This mixture was processed in a thermocycler at $94^{\circ} \mathrm{C}$ for $4 \mathrm{~min}$ (denaturation), followed by $30 \mathrm{cycles}$ at $94^{\circ} \mathrm{C}$ for $1 \mathrm{~min}$ (denaturation), annealing temperature (specific for each primer) for 1 min (binding), and at $72^{\circ} \mathrm{C}$ for $1.5 \mathrm{~min}$ (extension). Complete extension of the Taq DNA polymerase was performed at $72^{\circ} \mathrm{C}$ for $7 \mathrm{~min}$. An aliquot of this reaction containing only water without DNA was used as a negative control. Two multiplex PCR tests were carried out; one for the primer set LT, STa, and STb and the other for the primer set Stx 1 and Stx2.

\section{Serotyping}

The rabbit antisera against $\mathrm{O} 1$ to O187strains were prepared in rabbits (SERUNAM) according to procedures described by Ewing (31). The E. coli O1 to O172 strains were obtained from the Laboratory of Gastrointestinal Pathogens, Department of Gastrointestinal, Emerging and Zoonotic Infections, Centre for Infections, Health Protection Agency, London, UK, and the E. coli O173 to O186 strains (32) were obtained from the Statens Serum Institute, Copenhagen.

The strains were serotyped by agglutination assay (33) using 96-well micro titer plates and rabbit serum (SERUNAM) obtained against 187 somatic antigens and 53 flagellar antigens for E. coli.

\section{Antibiotic Susceptibility}

Susceptibility to antibiotics was performed by disk diffusion, as described by Bauer et al. (34) and the Clinical and Laboratory Standards Institute (35) (CLSI), using commercial antibiotic discs. Thirteen antibiotics (Oxoid, UK) were employed: erythromycin (E, $15 \mu \mathrm{g})$; gentamicin (CN, $10 \mu \mathrm{g})$; trimethoprim/ sulfamethoxazole (SXT, $30 \mu \mathrm{g}$ ), tetracycline (TE, $30 \mu \mathrm{g}$ ), ampicillin (AMP, $30 \mu \mathrm{g}$ ), streptomycin (S, $10 \mu \mathrm{g})$, azithromycin (AZM, $15 \mu \mathrm{g}$ ), nalidixic acid (NA, $30 \mu \mathrm{g}$ ), ciprofloxacin (CIP, $5 \mu \mathrm{g}$ ), ceftriaxone (CRO, $30 \mu \mathrm{g})$, cefixime (CFM, $5 \mu \mathrm{g}$ ), mecillinam (MEL, $25 \mu \mathrm{g}$ ), and cephalothin (KF, $30 \mu \mathrm{g}$ ). Characterizations of the resistance or susceptibility profiles of the isolates were determined by measuring the inhibitory zone and comparing it with an established interpretative chart to determine sensitivity to each antibiotic.

\section{Pulsed-Field Gel Electrophoresis (PFGE)}

Whole agarose-embedded genomic DNA from the E. coli isolates was prepared. PFGE was carried out using a contour-clamped homogeneous electrical field (CHEF-DRII) apparatus (Bio-Rad), according to procedures described previously (36). Genomic DNA of the test strains and Salmonella enterica serovar Braenderup was digested using $\mathrm{XbaI}$, with fragments employed as molecular size markers. Restriction fragments were separated in $1 \%$ pulsedfield-certified agarose in $0.5 \times$ TBE (Tris-borate-EDTA) buffer. Post-electrophoresis gel treatment included both gel-staining and de-staining. The DNA was visualized using a UV transilluminator, and images were digitized via a one-dimensional gel documentation system (Bio-Rad).

\section{PFGE Analysis}

The fingerprint pattern in the gel was analyzed using a computer software package, Bionumeric (Applied Maths, Belgium, version 3.1). After background subtraction and gel normalization, the fingerprint patterns were typed according to banding similarity and dissimilarity, using the Dice similarity coefficient and unweighted-pair group method employing average linkage (UPGMA) clustering, as recommended by the manufacturer. The results were graphically represented as dendrograms.

\section{RESULTS}

\section{Phenotypic Characteristics of E. coli}

All the E. coli strains $(n=35)$ included in this study produced bright pink colonies on MacConkey and green colonies with metallic sheen on EMB agar plates. All strains gave biochemical reactions typical of $E$. coli.

\section{Virulence Gene Profile}

Among the $35 \mathrm{E}$. coli strains isolated from livestock and poultry in the present study, 23 (66\%) (goats: 17, sheep: 4, chicken: 1, and ducks: 1) carried virulence genes, such as, sta and stx, either stx 1 or stx2, but did not carry stb or elt genes, as confirmed by PCR 
TABLE 2 | Serotypes, virulence gene, and drug resistance pattern of $E$. coli isolated from livestock samples in 2007 , Bangladesh.

\begin{tabular}{|c|c|c|c|c|c|c|c|c|}
\hline \multirow[t]{2}{*}{ No. of isolates } & \multicolumn{5}{|c|}{ Virulence genes } & \multirow[t]{2}{*}{ Serotype } & \multirow[t]{2}{*}{ Resistance pattern } & \multirow[t]{2}{*}{ Source } \\
\hline & Sta & Stb & $L t$ & Stx1 & Stx2 & & & \\
\hline 1 & + & - & - & - & - & O36:H5 & $E^{R}, A Z M^{R}$ & Goat \\
\hline 1 & + & - & - & + & - & O43:H2 & $E^{R}$ & Goat \\
\hline 2 & - & - & - & + & - & O43:H2 & $E^{R}$ & Goat \\
\hline 1 & + & - & - & + & - & O43:H2 & $E^{R}, A Z M^{R}, S^{R}$ & Goat \\
\hline 1 & - & - & - & + & - & O76:H19 & $E^{R}$ & Goat \\
\hline 1 & + & - & - & + & - & O76:H19 & $\mathrm{E}^{\mathrm{R}}, \mathrm{KF} \mathrm{F}^{\mathrm{R}}$ & Goat \\
\hline 1 & + & - & - & + & - & O76:H19 & Sensitive to all antibiotics & Goat \\
\hline 1 & - & - & - & + & - & O76:H19 & $E^{\mathrm{R}}$ & Goat \\
\hline 1 & + & - & - & + & - & O76:H19 & $E^{R}, A Z M^{R}, S^{R}$ & Goat \\
\hline 1 & - & - & - & + & - & O76:H19 & $\mathrm{E}^{\mathrm{R}}, \mathrm{AZM}^{\mathrm{R}}, \mathrm{S}^{\mathrm{R}}, \mathrm{K} \mathrm{F}^{\mathrm{R}}$ & Goat \\
\hline 1 & - & - & - & - & + & $\mathrm{O} 87: \mathrm{H} 16$ & $E^{\mathrm{R}}$ & Goat \\
\hline 1 & + & - & - & - & + & $\mathrm{O} 87: \mathrm{H} 16$ & $E^{R}, S X T^{R}, A Z M^{R}$ & Goat \\
\hline 1 & - & - & - & - & + & $\mathrm{O} 87: \mathrm{H} 16$ & $E^{R}, S X T^{R}, A Z M^{R}, S^{R}$ & Goat \\
\hline 1 & + & - & - & - & - & O174:H- & $E^{R}, S X T^{R}, A Z M^{R}, K F^{R}$ & Goat \\
\hline 1 & - & - & - & + & - & OR:H2 & $E^{\mathrm{R}}$ & Goat \\
\hline 1 & + & - & - & + & - & OR:H2 & $E^{R}, C I P^{R}, S X T^{R}, N A^{R}, A Z M^{R}, T E^{R}, K F^{R}$ & Goat \\
\hline 2 & - & - & - & - & - & ND & ND & Goat \\
\hline 1 & - & - & - & + & - & O110:H16 & $\mathrm{E}^{\mathrm{R}}$ & Sheep \\
\hline 1 & + & - & - & - & - & O152:H8 & $E^{R}, S^{R}$ & Sheep \\
\hline 1 & - & - & - & + & - & O152:H8 & Sensitive to all antibiotics & Sheep \\
\hline 1 & + & - & - & + & - & O152:H8 & Sensitive to all antibiotics & Sheep \\
\hline 5 & - & - & - & - & - & ND & ND & Sheep \\
\hline 2 & - & - & - & - & - & ND & ND & Cattle \\
\hline 1 & + & - & - & - & - & O109:H51 & $E^{R}, C_{P}^{R}, S X T^{R}, N A^{R}, A Z M^{R}$ & Chicken \\
\hline 2 & - & - & - & - & - & ND & ND & Chicken \\
\hline 1 & + & - & - & - & - & O8:H21 & $\mathrm{E}^{\mathrm{R}}, \mathrm{AZM}^{\mathrm{R}}$ & Duck \\
\hline 1 & - & - & - & - & - & ND & ND & Duck \\
\hline
\end{tabular}

E, erythromycin; AZM, azithromycin; S, streptomycin; KF, cephalothin; SXT, trimethoprim/sulfamethoxazole; CIP, ciprofloxacin; NA, nalidixic acid; TE, tetracycline; R, resistant; $N D$, not done.

(Table 2). Of the 23 strains carrying toxin genes, 5 (22\%) had sta, and $10(44 \%)$ had stx genes. Of the $10 \mathrm{E}$. coli carrying stx gene, $8(80 \%)$ harbored only $s t x 1$, and $2(20 \%)$ had only st 2 . Eight of the 23 (34\%) toxigenic strains were hybrid of which 7 (88\%) possessed both sta and $s t x 1$, while the remaining strain carried both sta and stx2 (Table 2). None of the strains tested presented elt or $s t b$.

\section{Serotyping of E. coli}

Based on serotyping, the 23 toxigenic E. coli strains belonged to 10 different $\mathrm{O}$ serogroups [O8 $(n=1), \mathrm{O} 36(n=1), \mathrm{O} 43(n=4)$, O76 $(n=6)$, O87 $(n=3)$, O109 $(n=1)$, O110 $(n=1), \mathrm{O} 152$ $(n=3), \mathrm{O} 174(n=1)$, and OR $(n=2)]$ and 8 different H seogroups $[\mathrm{H} 2(n=6), \mathrm{H} 5(n=1), \mathrm{H} 8(n=3), \mathrm{H} 16(n=4), \mathrm{H} 19$ $(n=6), \mathrm{H} 21(n=1), \mathrm{H} 51(n=1)$, and $\mathrm{H}-(n=1)]$. E. coli strains carrying virulence genes belonged to 10 different serotypes: O36:H5 $(n=1), \mathrm{O} 43: \mathrm{H} 2(n=4), \mathrm{O} 76: \mathrm{H} 19(n=6), \mathrm{O} 87: \mathrm{H} 16$ $(n=3), \mathrm{O} 174: \mathrm{H}-(n=1), \mathrm{OR}: \mathrm{H} 2(n=2), \mathrm{O} 110: \mathrm{H} 16(n=1)$, O152:H8 $(n=3)$, O109:H51 $(n=1)$, and O8:H21 $(n=1)$. None of the tested strains belonged to the EHEC serogroup, as they did not agglutinate with the antisera specific for O157 (Table 2). The ETEC strains $(n=5)$ belonged to five different serotypes: O36:H5, O174: H-, O152:H8, O109:H51, and O8:H21, while the STEC strains $(n=10)$ belonged to six different serotypes: O76:H19 (30\%), O43:H2 (20\%), O87:H16 (20\%), OR: H2 (10\%), O110:H16 (10\%), and O152:H8 (10\%) (Figure 1). In the order of prevalence of the STEC-ETEC hybrid strains $(n=8)$, O76:H19 was the most prevalent (37.5\%) serotype, followed by $\mathrm{O} 43: \mathrm{H} 2$ (25\%), O87:H16 (12.5\%), OR: H2 (12.5\%), and O152:H8 (12.5\%) (Figure 1).

\section{Antibiotic Assay}

Antibiotic susceptibility assay was performed for the 23 toxigenic strains only, and the results showed $87 \%$ of the strains to be resistant to erythromycin, $43 \%$ to azithromycin, $22 \%$ to streptomycin, $22 \%$ to trimethoprim/sulfamethoxazole, $17 \%$ to cephalothin, $9 \%$ to ciprofloxacin, $9 \%$ to nalidixic acid, and $4 \%$ to tetracycline (Table 2). All E. coli strains were sensitive to gentamicin, ampicillin, ceftriaxone, cefixime, and mecillinam. Results also revealed $40 \%(2 / 5)$ of the ETEC and $20 \%(2 / 10)$ of the STEC strains to be multidrug resistant (MDR) showing resistance to erythromycin, trimethoprim/sulfamethoxazole, azithromycin, cephalothin, ciprofloxacin, and nalidixic acid. The highest MDR was found in the hybrid strains, accounting for 50\% (4/8) (Table 2), although the strains varied in their patterns of response to the different drugs tested.

Overall drug response results revealed 11 different resistance patterns among the three pathogroups of E. coli (ETEC, STEC, and hybrids of STEC-ETEC). Resistance patterns I ( $\left.E^{\mathrm{R}}, \mathrm{S}^{\mathrm{R}}\right)$, II $\left(E^{\mathrm{R}}, \mathrm{AZM}^{\mathrm{R}}\right), \mathrm{III}\left(\mathrm{E}^{\mathrm{R}}, \mathrm{SXT}^{\mathrm{R}}, \mathrm{AZM}^{\mathrm{R}}, \mathrm{KF}^{\mathrm{R}}\right)$, and IV $\left(\mathrm{E}^{\mathrm{R}}, \mathrm{CIP}^{\mathrm{R}}, \mathrm{SXT}^{\mathrm{R}}\right.$, $\mathrm{NA}^{\mathrm{R}}, \mathrm{AZM}^{\mathrm{R}}$ ) were found in ETEC; resistance patterns $\mathrm{V}\left(\mathrm{E}^{\mathrm{R}}\right)$, VI $\left(E^{\mathrm{R}}, \mathrm{AZM}^{\mathrm{R}}, \mathrm{S}^{\mathrm{R}}, \mathrm{KF}^{\mathrm{R}}\right)$, and VII $\left(\mathrm{E}^{\mathrm{R}}, \mathrm{SXT}^{\mathrm{R}}, \mathrm{AZM}^{\mathrm{R}}, \mathrm{S}^{\mathrm{R}}\right)$ in STEC; and 


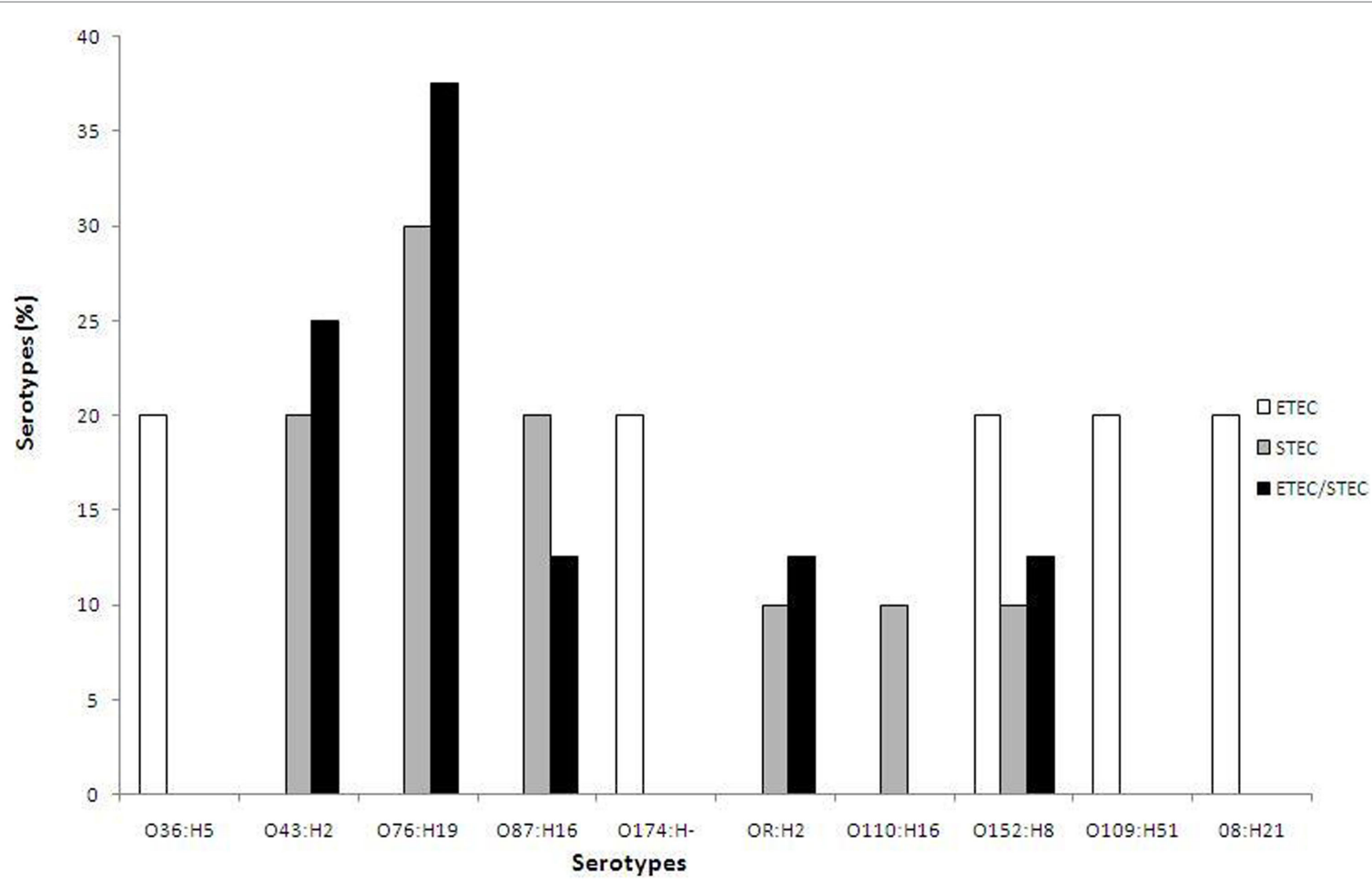

FIGURE 1 | Percentage of different serotypes of three E. coli pathogroups [enterotoxigenic E. coli (ETEC), STEC, and ETEC-STEC hybrid] from livestock animals (goat and sheep) and poultry (chicken and duck) in Bangladesh.

pattern V $\left(E^{\mathrm{R}}\right)$, VIII $\left(E^{\mathrm{R}}, \mathrm{KF}^{\mathrm{R}}\right), \mathrm{IX}\left(\mathrm{E}^{\mathrm{R}}, \mathrm{AZM}^{\mathrm{R}}, \mathrm{S}^{\mathrm{R}}\right), \mathrm{X}\left(\mathrm{E}^{\mathrm{R}}, \mathrm{SXT}^{\mathrm{R}}\right.$, $\left.\mathrm{AZM}^{\mathrm{R}}\right)$, and XI $\left(\mathrm{E}^{\mathrm{R}}, \mathrm{CIP}^{\mathrm{R}}, \mathrm{SXT}^{\mathrm{R}}, \mathrm{NA}^{\mathrm{R}}, \mathrm{AZM}^{\mathrm{R}}, \mathrm{TE}^{\mathrm{R}}, \mathrm{KF}^{\mathrm{R}}\right)$ among the STEC-ETEC hybrid pathotypes (Table 3).

\section{PFGE and Cluster Analysis}

The XbaI-digested genomic DNAs of the E. coli strains carrying virulence genes from animal and poultry were subjected to PFGE to determine genetic relatedness and clonal origin. The number of fragments generated by restriction digestion with $\mathrm{XbaI}$ varied between 14 and 19, and the molecular size of the fragments ranged from 30 to $600 \mathrm{~kb}$. Diverse PFGE patterns obtained from the 23 toxigenic E. coli strains (Figure 2) belonged to multiple small sub-clusters (Figure 2) when dendrograms were prepared with PFGE images by the BioNeumeric software (Applied Maths) using dice similarity coefficient and UPGMA. Although the E. coli strains exhibited high genetic heterogeneity in the PFGE patterns, four hybrid strains belonging to serotype O76:H19 formed a small tight cluster, suggesting that genetically, they are closely related.

\section{DISCUSSION}

\section{Occurrence of Hybrid of STEC and ETEC Strains}

Enterotoxigenic E. coli and Shiga toxin-producing E. coli (STEC) are notorious pathogens associated with severe diarrhea and
TABLE 3 | Antibiotic resistance profiles of the toxigenic E. coli $(n=23)$ isolates.

\begin{tabular}{|c|c|c|c|}
\hline Pathotype/virulence gene & Type & Antibiotic resistance profile & $\begin{array}{l}\text { No. of } \\
\text { strains }\end{array}$ \\
\hline \multirow{4}{*}{$\begin{array}{l}\text { Enterotoxigenic Escherichia } \\
\text { coli (ETEC) }\left(s t a^{+}, n=5\right)\end{array}$} & । & $E^{R}, S^{R}$ & 1 \\
\hline & $\|$ & $E^{R}, A Z M^{R}$ & 2 \\
\hline & III & $E^{R}, S X T^{R}, A Z M^{R}, K F^{R}$ & 1 \\
\hline & IV & $E^{R}, C^{R}, S X T^{R}, N A^{R}, A Z M^{R}$ & 1 \\
\hline \multirow[t]{4}{*}{ STEC $\left(s t \times 1^{+}\right.$or $\left.s t \times 2^{+}, n=10\right)$} & V & $E^{R}$ & 7 \\
\hline & $\mathrm{VI}$ & $E^{R}, A Z M^{R}, S^{R}, K F^{R}$ & 1 \\
\hline & VII & $E^{R}, S X T^{R}, A Z M^{R}, S^{R}$ & 1 \\
\hline & & Sensitive to all antibiotics & 1 \\
\hline \multirow{6}{*}{$\begin{array}{l}\text { STEC-ETEC hybrid }\left(s t a^{+} \text {and }\right. \\
\left.s t \times 1^{+} \text {or } s t \times 2^{+}, n=8\right)\end{array}$} & V & $E^{R}$ & 1 \\
\hline & VIII & $E^{R}, K F^{R}$ & 1 \\
\hline & IX & $E^{R}, A Z M^{R}, S^{R}$ & 2 \\
\hline & $x$ & $E^{R}, S X T^{R}, A Z M^{R}$ & 1 \\
\hline & $X I$ & $\begin{array}{l}E^{R}, C^{R}, S X T^{R}, N A^{R}, A Z M^{R}, \\
T^{R}, K F^{R}\end{array}$ & 1 \\
\hline & & Sensitive to all antibiotics & 2 \\
\hline
\end{tabular}

E, erythromycin; AZM, azithromycin; S, streptomycin; KF, cephalothin; SXT, trimethoprim/sulfamethoxazole; CIP, ciprofloxacin; NA, nalidixic acid; TE, tetracycline; $R$, resistant.

HUS in humans. Although E. coli constitutes an important component of normal gut bacterial flora, many studies have shown the non-toxigenic strains to harbor a number of different virulence factors and to be diarrheagenic $(17,18,37)$. Here, we present data showing the prevalence of $E$. coli strains carrying 


\section{Xba I}

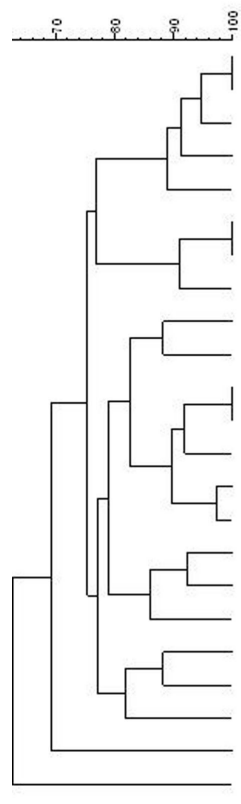

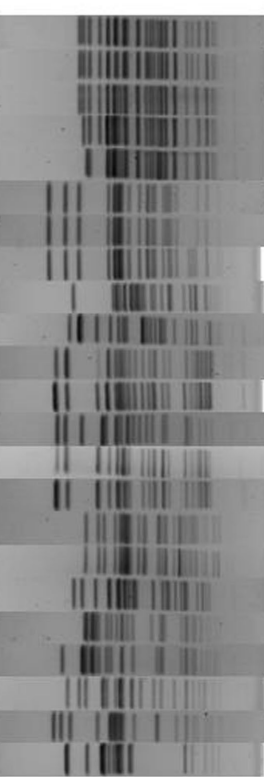

\section{Virulence gene profiles}

$\begin{array}{lllllll}\text { Source } & \text { sta } & \text { stb } & \text { It } & \text { stx1 } & \text { stX2 } & \text { Deduced Pathogroup } \\ \text { Goat } & + & - & - & + & - & \text { STEC-ETEC } \\ \text { Goat } & - & - & - & + & - & \text { STEC } \\ \text { Goat } & + & - & - & + & - & \text { STEC-ETEC } \\ \text { Goat } & + & - & - & + & - & \text { STEC-ETEC } \\ \text { Goat } & - & - & - & + & - & \text { STEC } \\ \text { Goat } & - & - & - & - & + & \text { STEC } \\ \text { Goat } & - & - & - & + & - & \text { STEC } \\ \text { Goat } & - & - & - & - & + & \text { STEC } \\ \text { Chicken } & + & - & - & - & - & \text { ETEC } \\ \text { Goat } & + & - & - & + & - & \text { STEC-ETEC } \\ \text { Goat } & - & - & - & + & - & \text { STEC } \\ \text { Goat } & - & - & - & + & - & \text { STEC } \\ \text { Goat } & + & - & - & - & + & \text { STEC-ETEC } \\ \text { Goat } & - & - & - & + & - & \text { STEC } \\ \text { Goat } & + & - & - & + & - & \text { STEC-ETEC } \\ \text { Goat } & + & - & - & - & - & \text { ETEC } \\ \text { Goat } & + & - & - & - & - & \text { ETEC } \\ \text { Goat } & + & - & - & + & - & \text { STEC-ETEC } \\ \text { Sheep } & - & - & - & + & - & \text { STEC } \\ \text { Sheep } & - & - & - & + & - & \text { STEC } \\ \text { Sheep } & + & - & - & - & - & \text { ETEC } \\ \text { Sheep } & + & - & - & + & - & \text { STEC-ETEC } \\ \text { Duck } & + & - & - & - & - & \text { ETEC }\end{array}$

FIGURE 2 | Pulsed-field gel electrophoresis (PFGE) patterns of Xbal-digested genomic DNA of potentially pathogenic $E$. coli strains ( $n$ = 23) isolated from different livestock's animal and poultry of Bangladesh in 2007. Serotypes, source, and virulence gene content are indicated. The dendrogram was prepared by the BioNeumeric software (Applied Maths) using dice similarity coefficient and unweighted-pair group method employing average linkage of the PFGE images of the E. coli strains. The scale bar at the top (left) indicates similarity coefficient (\%).

an ETEC-specific gene, sta, and STEC-specific gene, stx, which included either stx 1 or stx 2 in the gut of livestock and poultry in Bangladesh. This study also presents data showing for the first time that a significant proportion $(8 / 23)$ of the toxigenic $E$. coli strains from livestock were MDR and hybrid type carrying both stx (either stx 1 or stx2) and ETEC-specific enterotoxin gene, sta. The presence of such a high percentage of MDR hybrid type $E$. coli strains in livestock poses a potential health threat to people of Bangladesh.

\section{Virulence Gene Profiling of E. coli Strains}

Results obtained in this study confirmed that a very high proportion of the E. coli strains (66\%) deriving from different livestock are toxigenic, carrying either sta, stx, or both in varying combinations. What is most important to note is that a significant proportion of E. coli in livestock was confirmed to be ETEC, including $22 \%$ carrying the sta gene only, $44 \%$ STEC (harboring only stx gene; either stx 1 or st 2 ), and $35 \%$ hybrid comprising both est and stx. ETEC is an important cause of diarrhea in humans and animals in the low-income countries and is the main cause of diarrhea in travelers to low-income countries (1). One study carried out in Spain reported a high incidence of ETEC (30\%) in piglets with diarrhea (38). A study carried out in Bangladesh revealed $61 \%$ of the E. coli strains isolated from the aquatic environment carried the est gene (39). Zoonotic and environmental transmission may be the reason ETEC is the predominant group of $E$. coli associated with childhood diarrhea in Bangladesh (28). The occurrence of ETEC in farm animals has always been of great public health importance as animals excreting pathogenic bacteria can contaminate water bodies in and around the area they are reared and in many ways can be related to human diseases. This may be one reason why ETEC is largely a pathogen of low-income countries where access to safe drinking water is often low.

STEC strains can cause bloody diarrhea known as haemorrhagic colitis, non-bloody diarrhea, and HUS (1). The data presented in this study provide evidence that livestock is an important reservoir for STEC carrying either stx1 or stx2. A study carried out in Bangladesh reported $37.9 \%$ of buffalo, $20.1 \%$ of cows, and $10.0 \%$ of goats were positive for STEC (40). Our data appear to concur with $44 \%(n=10)$ of livestock E. coli isolated mostly from goats $(80 \%, 8 / 10$ or $35 \%, 8 / 23)$ revealed STEC, either stx1 or stx2. Our results also appear to agree with those from a study carried out in Vietnam that showed STEC to account for $27 \%$ of $E$. coli isolated from buffalos, $23 \%$ from cattle, and $38.5 \%$ from goats (41). Although water plays an important role in the transmission of pathogenic bacteria, a study carried out in Bangladesh reported the presence of stx only in $1.25 \%$ of the E. coli strains isolated from natural aquatic environments (39). STEC-associated diarrhea is also very low compared to that of other enteric pathogens, including Vibrio cholerae and ETEC in Bangladesh (27). This low prevalence of STEC as the etiology of diarrhea could be due to STEC-specific antibodies in the human population, as this pathotype is present in a high 
proportion of E. coli occurring in domesticated livestock, such as goats and sheep, although it was not found in poultry, and only rarely in surface water samples (39). The low prevalence in natural surface water could be related to the incompetence of the STEC strains to survive beyond their livestock reservoirs (cattle, sheep, and goats) (6). Despite the low prevalence of STEC infections in Bangladesh (27), the virulence potential of these livestock strains to emerge as a novel STEC pathogen cannot be ruled out.

In the present study, $34 \%$ of the toxigenic livestock E. coli strains were hybrid as those carried toxin genes present in two different pathotypes; stx and est. Notably, majority of these hybrid strains $(7 / 8)$ carried both sta and stx 1 , while one strain carried both sta and st $x 2$ genes. This finding is not unique to our study as a number of past studies have shown hybrid strains carrying virulence marker genes of two different $E$. coli pathotypes (17), nonetheless, in a significantly lower frequency (2\%) than that we observed in Bangladesh. Clinically linked STEC-ETEC hybrid E. coli strains from Brazil, Denmark, France, Germany, Great Britain, Mexico, and the United States were shown to harbor virulence markers for two or three different pathotypes (17). In a study in Germany, $0.6 \%$ of the human STEC isolates possessed stx $2 g$ and estIa (37). Serotype O100:H of a STEC-ETEC hybrid strain carrying stx2 and estIa genes was isolated from contaminated drinking water during an outbreak in Finland (22). A later study reported a STEC strain carrying stx $1 a$ and estIa from cattle in Burkina Faso (42). Although no data exist on the virulence potential of STEC-ETEC hybrid strains isolated from livestock in Bangladesh, the widespread distribution and clinical relevance worldwide might indicate their virulence potential.

\section{Serodiversity}

In the present study, serotyping results revealed that the ETEC strains belonged to five different serotypes: O36:H5, O174:H-, O152:H8, O109:H51, and O8:H21. No sero-specificity was observed for these ETEC strains as there was no relationship between the serotypes and the type of toxin genes (st or $l t$ ) they possessed. Likewise, the STEC strains belonged to six different serotypes (O76:H19, O43:H2, O87:H16, OR: H2, O110:H16, and O152:H8); STEC O157:H7 serotype was not present among the tested strains. Moreover, the STEC serotypes did not belong to any of the serotypes of the big six groups (O26, O45, O103, O111, O121, and O145) in the present study (43). STEC non-O157 isolates have been shown to be important pathogens despite having been underreported because in many laboratories, the facilities to isolate, identify, and characterize this pathotype do not exist (40). One report suggested that some non-O157:H7 STEC strains cause human illness and likely account for $20-50 \%$ of STEC infections (an estimated 37,000 cases) annually in the United States (44). In our study, we have identified eight E. coli strains that were hybrids and carried virulence genes STECETEC, which are largely restricted to five serotypes (O76:H19, O43:H2, O87:H16, OR: H2, and O152:H8). Several other studies reported STEC-ETEC hybrid strains; however, unlike our study, the serotypes were O2:H27, O101:H-, O15:H16, O74:H28, O116:H28, O128:H8, O136:H12, O141:H8, O168:H8,
OX182:H16, OX182:H- in Finland (23), O139, O149, O116, and OSB9 in Japan (24), O100:H- in United States (21), and O2:H2 in Burkina Faso (42).

\section{Antibiotic Resistance Profile}

Resistance to antimicrobial agents can be a useful epidemiological marker for ETEC, STEC, and STEC-ETEC hybrid strains. In this study, the highest antimicrobial resistance was found against erythromycin $87 \%(20 / 23)$ followed by azithromycin $43 \%$ $(10 / 23)$. In the present study, a significant proportion of ETEC and STEC strains were MDR with the highest MDR [50\% (4/8)] being observed in STEC-ETEC hybrid strains. Since 1950s, antibiotics have been used in animal feed to ensure healthy livestock, prevent infections, and excel growth. Such indiscriminate use of antibiotics resulted in the widespread antibiotic resistance in bacteria (21). Erythromycin resistance during the 1990s compelled clinicians to switch to azithromycin as the drug of choice in the treatment of cholera (45) and watery (cholera-like) diarrhea caused by ETEC $(46,47)$.

\section{PFGE Analysis}

As shown earlier by $\mathrm{Vu}-\mathrm{Khac}$ et al. (48), a high degree of genetic polymorphisms was observed from the PFGE profiles of the STEC non-O157 and ETEC strains in the present study. Similarly, a high degree of heterogeneity was also observed with the STEC-ETEC hybrid strains, as the pulsotypes were different for strains belonging to the same serotypes, suggesting that they were unlikely to be of a single ancestral origin. A few strains showing identical PFGE patterns, however, suggest close clonal relatedness.

\section{CONCLUSION}

This study is the first to show livestock as the reservoir for multidrug resistant hybrid type E. coli strains carrying both stx and st found in Shiga toxin-producing E. coli (STEC) and ETEC, respectively, in Bangladesh. Serotyping results revealed that none of the E. coli STEC-ETEC hybrid strains were O157:H7 but belonged to serotypes O76:H19, O43:H2, O87:H16, OR:H2, and O152:H8 and were heterogeneous genetically as confirmed by PFGE of XbaI-digested genomic DNA and cluster analyses by dendrogram. Although there may be some sorts of immunity toward Shiga toxin-producing E. coli existing among the healthy human population of Bangladesh (49), as they live in close proximity to the domesticated animals, multidrug resistant STEC-ETEC hybrid E. coli strains occurring in domesticated animals deserve careful attention as they can pose even greater public health threat for the people of Bangladesh.

\section{AUTHOR CONTRIBUTIONS}

AK, MO, HW, and MA contributed to the design of the study, manuscript revision, and final approval of version to be published. F-TJ designed, implemented, and performed the study in the laboratory and wrote the manuscript. SC, CG, AC, SM, SA, $\mathrm{BH}$, and $\mathrm{AN}$ contributed to revising the manuscript critically for 
important intellectual content. RP, AI, AS, and MR performed the study in the laboratory. All the authors read and approved the final manuscript.

\section{ACKNOWLEDGMENTS}

icddr,b gratefully acknowledges the following donors for providing unrestricted support: Governments of the People's Republic of Bangladesh, Global Affairs Canada (GAC), Swedish

\section{REFERENCES}

1. Kaper JB, Nataro JP, Mobley HLT. Pathogenic Escherichia coli. Nat Rev Microbiol (2004) 2:123-40. doi:10.1038/nrmicro818

2. Levine MM. Escherichia coli that cause diarrhea: enterotoxigenic, enteropathogenic, enteroinvasive, enterohemorrhagic, and enteroadherent. J Infect Dis (1987) 155(3):377-89. doi:10.1093/infdis/155.3.377

3. Nataro JP, Kaper JB. Diarrheagenic Escherichia coli. Clin Microbiol Rev (1998) 11:142-201.

4. Ørskov F, Ørskov I. Escherichia coli serotyping and disease in man and animals. Can J Microbiol (1992) 38:699-704. doi:10.1139/m92-115

5. Tarr PI, Gordon CA, Chandler WL. Shiga-toxin-producing Escherichia coli and haemolytic uraemic syndrome. Lancet (2005) 365:1073-86. doi:10.1016/ S0140-6736(05)71144-2

6. Griffin PM, Tauxe RV. The epidemiology of infections caused by Escherichia coli $\mathrm{O} 157: \mathrm{H} 7$, other enterohemorrhagic E. coli, and the associated hemolytic uremic syndrome. Epidemiol Rev (1991) 13:60-98.

7. Blanco M, Blanco JE, Mora A, Dahbi G, Alonso MP, González EA, et al. Serotypes, virulence genes, and intimin types of Shiga toxin (verotoxin)producing Escherichia coli isolates from cattle in Spain and identification of a new intimin variant gene (eae $)$. J Clin Microbiol (2004) 42:645-51. doi:10.1128/JCM.42.2.645-651.2004

8. Luna-Gierke RE, Griffin PM, Gould LH, Herman K, Bopp CA, Strockbine $\mathrm{N}$, et al. Outbreaks of non-O157 Shiga toxin-producing Escherichia coli infection: USA. Epidemiol Infect (2014) 142(11):2270-80. doi:10.1017/ S0950268813003233

9. Pradel N, Livrelli V, Champs CDe, Palcox JB, Reynaud A, Scheutz F, et al. Prevalence and characterization of Shiga toxin-producing Escherichia coli isolated from cattle, food, and children during a one-year prospective study in France. J Clin Microbiol (2000) 38:1023-31.

10. Izumikawa K, Hirakata Y, Yamaguchi T, Yoshida R, Nakano M, Matsuda J, et al. Analysis of genetic relationships and antimicrobial susceptibility of Verotoxin-producing Escherichia coli strains isolated in Nagasaki Prefecture, Japan in 1996. Microbiol Immunol (1998) 42:677-81. doi:10.1111/j.1348-0421. 1998.tb02339.x

11. Nagy B, Fekete PZ. Enterotoxigenic Escherichia coli (ETEC) in farm animals. Vet Res (1999) 30:259-84.

12. Ngeleka M, Pritchard J, Appleyard G, Middleton DM, Fairbrother JM. Isolation and association of Escherichia coli AIDA-I/STb, rather than EAST1 pathotype, with diarrhea in piglets and antibiotic sensitivity of isolates. $J$ Vet Diagn Invest (2003) 15:242-52. doi:10.1177/104063870301500305

13. Lortie LA, Dubreuil JD, Harel J. Characterization of Escherichia coli strains producing heatstable enterotoxin $\mathrm{b}(\mathrm{STb})$ isolated from humans with diarrhea. J Clin Microbiol (1991) 29:656-9.

14. Furrer B, Candrian U, Luthy J. Detection and identification of E. coli producing heat-labile enterotoxin type I by enzymatic amplification of a specific DNA fragment. Lett Appl Microbiol (1990) 10:31-4. doi:10.1111/j.1472-765X.1990. tb00088.x

15. Jackson MP, Neill RJ, O'Brien AD, Holmes RK, Newland JW. Nucleotide sequence analysis and comparison of the structural gene for Shiga-like toxin I and Shiga-like toxin II encoded by bacteriophages from Escherichia coli 933. FEMS Microbiol Lett (1987) 44:109-14. doi:10.1111/j.1574-6968.1987. tb02252.x

16. Jackson MP, Newland JW, Holmes RK, O’Brien AD. Nucleotide sequence analysis of the structural genes for Shiga-like toxin I encoded by
International Development Cooperation Agency (Sida), and the Department for International Development (UK Aid).

\section{FUNDING}

MA acknowledges NIH grant \#R01AI039129 for supporting him. This research study was supported jointly by icddr,b, University of Dhaka, and the National Institutes of Infectious Diseases (NIID), Japan.

bacteriophage 933J from Escherichia coli. Microb Pathog (1987) 2:147-53. doi:10.1016/0882-4010(87)90106-9

17. Müller D, Greune L, Heusipp G, Karch H, Fruth A, Tschape H, et al. Identification of unconventional intestinal pathogenic Escherichia coli isolates expressing intermediate virulence factor profiles by using a novel single-step multiplex PCR. Appl Environ Microbiol (2007) 73:3380-90. doi:10.1128/ AEM.02855-06

18. Mellmann A, Harmsen D, Cummings CA, Zentz EB, Leopold SR, Rico A, et al. Prospective genomic characterization of the German enterohemorrhagic Escherichia coli O104:H4 outbreak by rapid next generation sequencing technology. PLoS One (2011) 6:e22751. doi:10.1371/journal.pone. 0022751

19. Bielaszewska M, Mellmann A, Zhang W, Kock R, Fruth A, Bauwens A, et al. Characterisation of the Escherichia coli strain associated with an outbreak of haemolytic uraemic syndrome in Germany, 2011: a microbiological study. Lancet Infect Dis (2011) 11:671-6. doi:10.1016/S1473-3099(11) 70165-7

20. Vu-Khac H, Holoda E, Pilipcinec E, Blanco M, Blanco JE, Dahbi G, et al. Serotypes, virulence genes, intimin types and PFGE profiles of Escherichia coli isolated from piglets with diarrhoea in Slovakia. Vet J (2007) 174:176-87. doi:10.1016/j.tvjl.2006.05.019

21. Fratamico PM, Bhagwat AA, Injaian L, Fedorka-Cray PJ. Characterization of Shiga toxin-producing Escherichia coli strains isolated from swine feces. Foodborne Pathog Dis (2008) 5:827-38. doi:10.1089/fpd.2008.0147

22. Lienemann T, Pitkanen T, Antikainen J, Molsa E, Miettinen I, Haukka K, et al. Shiga toxin producing Escherichia coli O100:H-:stx2e in drinking water contaminated by waste water in Finland. Curr Microbiol (2011) 62:1239-44. doi:10.1007/s00284-010-9832-x

23. Nyholm O, Heinikainen S, Pelkonen S, Hallanvuo S, Haukka K, Siitonen A. Hybrids of shigatoxigenic and enterotoxigenic E. coli (STEC/ETEC) among human and animal isolates in Finland. Zoonoses Public Health (2015) 62:518-24. doi:10.1111/zph.12177

24. Kusumoto M, Hikoda Y, Fujii Y, Murata M, Miyoshi H, Ogura Y, et al. Emergence of a multidrug-resistant Shiga toxin-producing enterotoxigenic $E$. coli lineage in diseased swine in Japan. J Clin Microbiol (2016) 54(4):1074-81. doi:10.1128/JCM.03141-15

25. Leonard SR, Mammel MK, Rasko DA, Lacher DW. Hybrid Shiga toxin-producing and enterotoxigenic Escherichia sp. cryptic lineage 1 strain 7v harbors a hybrid plasmid. Appl Environ Microbiol (2016) 82(14):4309-19. doi:10.1128/ AEM.01129-16

26. EFSA. Urgent advice on the public health risk of Shiga toxin producing Escherichia coli in fresh vegetables. EFSA J (2011) 9:2274. doi:10.2903/ j.efsa.2011.2274

27. Islam MA, Heuvelink AE, de Boer E, Sturm PD, Beumer RR, Zwietering $\mathrm{MH}$, et al. Shiga toxin-producing Escherichia coli isolated from patients with diarrhea in Bangladesh. J Med Microbiol (2007) 56:380-5. doi:10.1099/ jmm.0.46916-0

28. Qadri F, Svennerholm AM, Faruque AS, Sack RB. Enterotoxigenic Escherichia coli in developing countries: epidemiology, microbiology, clinical features, treatment and prevention. Clin Microbiol Rev (2005) 18:465-83. doi:10.1128/ CMR.18.3.465-483.2005

29. Feng P, Weagant SD, Grand MA. Enumeration of Escherichia coli and the coliform bacteria. Bacteriological Analytical Manual Online. Chapter 4. (2002). Available from: http://www.fda.gov/Food/FoodScienceResearch/ LaboratoryMethods/ucm064948.htm 
30. Keskimaki M, Eklund M, Personen H, Heiskanen T, Siitonen A, Study Group. EPEC, EAEC and STEC in stool specimens: prevalence and molecular epidemiology of isolates. Diagn Microbiol Infect Dis (2001) 40:151-6. doi:10.1016/ S0732-8893(01)00265-6

31. Ewing WH. Edwards and Ewing's Identification of Enterobacteriaceae. New York: Elsevier (1986)

32. Scheutz F, Cheasty T, Woodward D, Smith HR. Designation of O174 and O175 to temporary O groups OX3 and OX7, and six new E. coli O groups that include verocytotoxin producing E. coli (VTEC): O176, O177, O178, O179, O180 and O181. APMIS (2004) 112:569-84. doi:10.1111/j.1600-0463.2004. apm1120903.x

33. Ørskov F, Ørskov I. Serotyping of Escherichia coli. In: Bergan T, editor. Methods in Microbiology (Vol. 14), London: Academic Press (1984). p. 43-112.

34. Bauer AW, Kirby WM, Sheris JC, Turck M. Antibiotics susceptibility testing by standardized single disk method. Am JClin Pathol (1966) 1966(45):493-6.

35. Clinical and Laboratory Standards Institute. Performance Standards for Antimicrobial Disk Susceptibility Tests; Approved Standard-11 $1^{\text {th }}$ Edition, Document M02-A11. Wayne, PA: Clinical and Laboratory Standards Institute (2012).

36. Ribot EM, Fair MA, Gautom R, Cameron DN, Hunter SB, Swaminathan B, et al. Standardization of pulsed-field gel electrophoresis protocols for the subtyping of Escherichia coli O157:H7, Salmonella, and Shigella for PulseNet. Foodborne Pathog Dis (2006) 3:59-67. doi:10.1089/fpd.2006.3.59

37. Prager R, Fruth A, Busch U, Tietze E. Comparative analysis of virulence genes, genetic diversity, and phylogeny of Shiga toxin $2 \mathrm{~g}$ and heat-stable enterotoxin STIa encoding Escherichia coli isolates from humans, animals, and environmental sources. Int J Med Microbiol (2011) 301(3):181-91. doi:10.1016/ j.ijmm.2010.06.003

38. Blanco J, Blanco M, Garabal JI, Gonzalez EA. Enterotoxins, colonization factors and serotypes of enterotoxigenic Escherichia coli from humans and animals. Microbiologia (1991) 7:57-72.

39. Alam M, Hasan NA, Ahsan S, Pazhani P, Tamura K, Ramamurthy T, et al. Phenotypic and molecular characterization of Escherichia coli isolated from aquatic environment of Bangladesh. Microbiol Immunol (2006) 50(5):359-70. doi:10.1111/j.1348-0421.2006.tb03802.x

40. Islam MA, Mondol AS, Boer ED, Beumer RR, Zwietering MH, Talukder KA, et al. Prevalence and genetic characterization of Shiga toxin-producing Escherichia coli isolates from slaughtered animals in Bangladesh. Appl Environ Microbiol (2008) 74:5414-21. doi:10.1128/AEM.00854-08

41. Vu-Khac H, Cornick NA. Prevalence and genetic profiles of Shiga toxinproducing Escherichia coli strains isolated from buffaloes, cattle, and goats in central Vietnam. Vet Microbiol (2008) 126:356-63. doi:10.1016/ j.vetmic.2007.07.023

42. Martikainen O, Kagambega A, Bonkoungou IJ, Barro N, Siitonen A, Haukka K. Characterization of shigatoxigenic Escherichia coli strains from Burkina Faso. Foodborne Pathog Dis (2012) 9:1015-21. doi:10.1089/fpd.2012.1228

43. Brooks JT, Sowers EG, Wells JG, Greene KD, Griffin PM, Hoekstra RM, et al. Non-O157 Shiga toxin-producing Escherichia coli infections in the United States, 1983-2002. J Infect Dis (2005) 192:1422-9. doi:10.1086/466536

44. Mead PS, Slutsker L, Dietz V, McCaig LF, Bresee JS, Shapiro C, et al. Foodrelated illness and death in the United States. Emerg Infect Dis (1999) 5:607-25. doi:10.3201/eid0505.990502

45. Bhattacharya MK, Dutta D, Ramamurthy T, Sarkar D, Singharoy A, Bhattacharya SK. Azithromycin in the treatment of cholera in children. Acta Paediatr (2003) 92:676-8. doi:10.1111/j.1651-2227.2003.tb00598.x

46. Burans JP, Podgore J, Mansour MM, Farah AH, Abbas S, Abu-Elyazeed R, et al. Comparative trial of erythromycin and sulphatrimethoprim in the treatment of tetracycline-resistant Vibrio cholerae O1. Trans R Soc Trop Med Hyg (1989) 83(6):836-8. doi:10.1016/0035-9203(89)90347-7

47. Saha D, Karim MM, Khan WA, Ahmed S, Salam MA, Bennish ML. Singledose azithromycin for the treatment of cholera in adults. N Engl J Med (2006) 354(23):2452-62. doi:10.1056/NEJMoa054493

48. Vu-Khac H, Holoda E, Pilipcinec E, Blanco M, Blanco JE, Mora A, et al. Serotypes, virulence genes, and PFGE profiles of Escherichia coli isolated from pigs with postweaning diarrhoea in Slovakia. BMC Vet Res (2006) 2:10. doi:10.1186/1746-6148-2-10

49. Mirza N. Immune Response to Shiga Toxin Producing Escherichia coli: Detection of Antibodies against Outer Membrane Proteins of the Bacteria in Healthy Population of Bangladesh. MS thesis. Brac University, Dhaka, Bangladesh (2015).

Conflict of Interest Statement: The authors declare that the research was conducted in the absence of any commercial or financial relationships that could be construed as a potential conflict of interest.

Copyright (c) 2017 Johura, Parveen, Islam, Sadique, Rahim, Monira, Khan, Ahsan, Ohnishi, Watanabe, Chakraborty, George, Cravioto, Navarro, Hasan and Alam. This is an open-access article distributed under the terms of the Creative Commons Attribution License (CC BY). The use, distribution or reproduction in other forums is permitted, provided the original author(s) or licensor are credited and that the original publication in this journal is cited, in accordance with accepted academic practice. No use, distribution or reproduction is permitted which does not comply with these terms. 